\title{
CAN YOU T.E.A.C.H. ADOLESCENTS?
}

Kamberk-Kilicci M. (University of Montreal, Montreal, Canada) mary.kilicci@gmail.com útrlumjugvimi mú. ' 30.06.2019

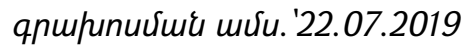

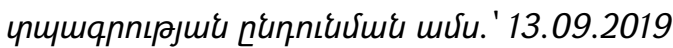

The main question underlying this study refers to how teachers may be helped to address the psychological factors associated with the well-being of their adolescent students. In the realm of professional development, the teachers are offered to review their background knowledge about the psychology of adolescents and use what research and experience have taught to support, encourage and enhance their students' learning. The adolescent is obviously central to the equation with his/her typical characteristics of increased detachment from adults, overall emotional vulnerability, the emphasis on the peer group and the essential search for identity. The second refrain has to do with the various roles of the teacher as a person, what he/she needs to know and how he/she needs to be in order to provide his/her students with the opportunities to learn in ways that meet their various needs. Finally, the third and last chapter elaborates on what T.E.A.C.H. means: T for thought, $\mathrm{E}$ for empathy; A for activity; $\mathrm{C}$ for choice and $\mathrm{H}$ for harmony. It also provides a discussion about how each aspect evolves with time and integrates within the scope of research relative to the domain of teaching and psychology.

Keywords: adolescence, search for identity, teaching, empathy, trust.

"We all need encouragement. We can live without it just as a young tree can live without fertilizer, but unless we receive that warm nurturing, we never reach our full potential. And like the tree left to itself, we seldom bear fruit." Florence Littauer

This professional development review will help you know more about adolescents and about your role as their teacher. It is divided into three parts: The adolescent, the role of the teacher, what T.E.A.C.H. means.

THE ADOLESCENT: Adolescents are in search of their identity. In the process, they become increasingly detached from adults and get closer to peers. Most are emotionally vulnerable, and a long-term perspective is beneficial in helping them cross the bridge in becoming adults.

The search for Identity: The search for identity is described by Erikson (1968) as life's fifth psychosocial conflict: identity versus diffusion or role confusion. It is about the need that adolescents have to determine who they are, their own identity. It's about their challenge to become responsible and independent adults. Generally, they do this by questioning the customs, beliefs, and values imposed on them by adults so they can 
break free and choose for themselves. In most cases, adjustment comes gradually. When the opposite happens, and an adolescent fails to discover his or her identity, he or she may be diffused, that is, confused about his/her goals in life, such as finishing school, finding a job, or thinking about the future. Such adolescents may fall into a state of indifference and find it difficult to fulfill all sorts of responsibilities. They may develop a who cares or what does it matter attitude where the slightest effort to achieve a goal may be seen as futile.

According to Erikson (1963), during the many years of adolescence, the problem is not so much the experience of confusion but its intensity and duration. In other words, numerous new experiences may lead to temporary states of confusion, but these are usually short-lived, and adolescents end up finding a way out.Today it is generally agreed that Erikson's theory, though half a century old, is still universally valid, but some factors involving its application vary from culture to culture and family to family. For example, certain cultures encourage independence and accelerate the search for identity, while others delay its onset through overprotection. Overall, most adolescents do end up being responsible adults who choose careers, find a partner, and settle clown. They may also choose to remain connected to parental values, fully or partially.

Increased Detachment from Adults: In searching for their own identity, young people normally tend to detach themselves by developing tastes and preferences that are unique and different from those of the adult world, such as the music they listen to, the clothes they wear, the hairstyles they choose, and the type of social gatherings they enjoy. In essence, the way adults respond to this may make the generation gap smaller or greater. Supportive adults can help adolescents feel more valued, trusted, and accepted, and therefore adolescents may not feel as much need for distance. However, when highly traditional parental values and standards are imposed, the gap between generations is bound to increase.

The Importance of Peers: During adolescence peers play a crucial role providing mutual trust, respect, and support in the passage from childhood to independence. Peer relationships provide a setting where adolescents explore all kinds of social skills. Their role can be visualized in the form of metaphors such as:

a) a mirror: one adolescent serves as a mirror for another, as no one is better than a peer to reflect the adolescent's own wishes, doubts, anxieties, dreams, and frustrations.

b) a walking stick: may serve to lean on at moments of stress and anxiety, such as when studying for exams, practicing for a competition, going through family-related stress, etc.

c) a magnet: for better or worse. High achievers attract others who are also ambitious academically and success-oriented, whereas troubled adolescents attract others who engage in high-risk behavior such as cheating and dangerous activities like 
speeding, using drugs or alcohol, etc. These metaphors illustrate how adolescents use peers to get to know themselves and to evaluate their strengths and weaknesses. The true markers of trust and respect no longer come from adults but from peers. In fact, no other criteria can replace the power of the peer groups' criteria when it comes to choosing clothes, activities, tastes in food, music or games. The peer group offers valuable protection for an adolescent who is emotionally vulnerable. It provides numerous learning experiences and opportunities to develop same-sex as well as opposite-sex friendships. Adolescents may find in the peer group an anchor, a secure place, a nest... This can give them a feeling of belonging and a sense of trust. It can provide a setting in which to learn from their own mistakes. It can also provide support to the adolescent in cases of serious conflict with authority. Finally, through exchanges and discussions with peers, a lot of learning takes place. As ideas are exchanged and points of views clash, adolescents realize how vulnerable they can be. This realization is valuable as it gradually takes them away from their egocentrism to a more realistic vision of themselves and others.

Emotional Vulnerability: F. Dolto (1997), the well-known French psycho-analyst, talks about the vulnerability of adolescents. She uses the metaphor of the lobster that bas lost its shell and hides under rocks in deep waters, waiting for a new protective covering to form. She states that during this time, adolescents feel defenseless against any type of attack and if they get hurt, the scars may not disappear but remain under their new shell.

Intellectually, adolescents are capable of the type of abstract thinking associated with a higher level of reasoning rather than the concrete logic of the elementary schoolage child (J. Piaget, 1966). Based on their superior thinking abilities, adolescents are well equipped to reason about ideas, attitudes, rules, beliefs, customs, and values. If this is the case, then why are they so vulnerable? One reason is that, despite their physiological maturity and their advanced ability to think and to reason, adolescents are still intellectually as well as emotionally rather immature. In fact, they are self-centered or egocentric although in a different way from that of the earlier years.

Another aspect of the vulnerability of adolescents is reflected in their feeling of "uniqueness". Elkind (1979) explains this feeling using the analogy of being "on stage" and being watched by an "imaginary audience". Interestingl, vandalism is considered to be an extension (pathological) of imaginary audience behavior. In destroying the property of others for no rational reason, vandals, who are often adolescents, may imagine the victims' reactions to the damage caused (windows broken, things stolen, sometimes in school settings). Finally, together with adolescent egocentrism, comes magical thinking. This type of thinking is inconsistent with the advanced reasoning abilities of adolescents. Having distanced themselves from adults, having de-idealized them, it's as though adolescents now need to dream in order to satisfy their need for an unlimited degree of power. Magical thinking is reflected 
in all forms of expression, such as language, poems, writings, and songs. It does not have to respect the boundaries of logic. For example, adolescents can see themselves as "reformers" or "saviors" with the ability to solve the world's problems or to create an imaginary utopia. One should note however that the intensity of such attitudes is highly dependent on the age of the adolescent (i.e. physiological and sexual changes in the body), his or her personal vulnerability as well as the social and cultural standards of the surrounding society.

THE ROLE OF THE TEACHER: Knowing the developmental characteristics of their students, teachers can do their best to adopt an attitude that combines empathy, a willingness to inspire trust and respect, and a readiness to communicate. They can set and apply firm limits while also being able to reach a compromise when necessary.

Teachers should be Empathetic: Empathy is a term that refers specifically to a person's growing ability to understand and respond to the inner feelings of others. In the classroom, an empathetic teacher may be willing to express concern even when a student behaves in a way that is annoying. She or he tries to understand the reason behind the student's misbehavior by putting herself or himself in the student's shoes for a moment. In fact, a genuine understanding of the feelings and concerns of the students is essential in teaching adolescents because it brings the students closer to the teacher rather than distancing them. According to various research studies, empathy works in the opposite way from anger, dislike, aggression, and other anti-social behaviors while leading to pro-social behavior: sharing, caring, and generosity (Mussen, P., EisenbergBerg, N. 1977). The teacher who is empathetic can obtain the cooperation of her/his students more easily and hence reinforce behaviors that create a positive learning environment in the classroom.

Teachers Should Inspire Trust and Respect: The teacher's willingness to inspire the trust of the students is essential for better learning. Trust, being a basic and integral part of psychosocial development, is primary to all meaningful relationships in life. In any social interaction, when trust and security are missing, the individual cannot engage confidently in exploration in order to grow and develop (Erikson, 1963). A trusting relationship, on the other hand, motivates individuals to fulfill their potential.

The benefits of developing a trusting relationship between student and teacher cannot be emphasized enough. In reality, it can have a major impact on the classroom climate and prevent behavioral problems. Note that a trusting relationship happens when the teacher knows the student's needs, takes the time to observe, listen and display empathy while being consistent and coherent in applying rules and regulations.

Finally, note that it is important to seek the help of professionals in order to feel reassured; but teachers should not be naïve enough to think that solutions are to be found outside rather than inside the classroom. Teachers need to believe, 
through (a) increased knowledge about their students and (b) reduced apprehension of their students, that they have a lot more to give than merely transmitting the specific subject they teach.

Teachers should be Open to Dialogue and Exchange Ideas: Open and communicative teachers address the need of adolescents to interact socially with each other on a regular basis. Moreover, because adolescents have a natural tendency to distance themselves from adults, the open teacher accepts more readily the adolescents' need to question rules and regulations, to impose their own points of view and to be somewhat self-centered.

The Need to Set Firm Limits and Apply Them: To prevent possible behavioral problems in the classroom and to ensure better learning, teachers need to recognize and use both sides of the coin: on one side having an open, respectful, and accepting attitude, and on the other, setting and applying firm limits. Based on our knowledge of the typical adolescent, it follows that students should be invited to participate in setting the rules for proper behavior in the classroom. However, doing this does not guarantee that they will necessarily follow these rules. Not many adolescents have self-discipline. Not all, as children, have had similarly positive learning experiences in their elementary school years and not all may have developed the self-reliance that enables them to behave properly in different social situations. All teachers, whatever their approach to teaching or their social and cultural beliefs and values, in order to maintain a peaceful atmosphere for learning, may benefit from defining precise rules and regulations for appropriate behavior. Teachers need to present positive role models by respecting the limits that they set and apply in a consistent fashion and in line with the values they want to convey to their students (i.e. being supportive of each other, accept differences, consider the needs and motives of others, etc...) This way, they may be able to instill more trust in their students.

Teachers Should Show the Will to Compromise: Of all teacher qualities, compromise is perhaps the most important. Before getting deeper into the characteristics that it entails for the teacher, it is interesting to see that compromise is associated with a balance between give and take. Compromise has an essential place in social development and positive social skills. It is associated with cooperation among individuals and is not compatible with self-centered behaviors.

Adolescent misbehavior is often the result of adolescents' greater need for power than they are ready to assume. Rigid rules and regulations, when applied without any consideration for the adolescent's need for power, increase the gap between teachers and students. This is the opposite of what the student requires in order to establish trust. Therefore, a certain degree of flexibility is important. A teacher who has high self-esteem can be a better negotiator by being ready to show firmness but at the same time flexibility. 
THE FIVE ELEMENTS OF T. E. A. C. H.: Based on the psychological knowledge provided above, the letters T.E.A. C. H. may define five essential elements for teachers to remember.

T for thought: Thinking opens the door to better learning and personal development. Teachers should remember that adolescents have the potential for advanced reasoning, despite the fact that they are often overwhelmed by intense emotions. Therefore teachers need to make an extra effort to invite their students to reflect on the way they or their classmates behave. Thinking is associated with problem solving, which increases students' self-confidence and autonomy. Thinking something over helps students reflect upon the motives behind their behavior and as such, is associated with self-control, which takes into consideration the needs of others as well as one's own. Thinking behavior enhances creativity and the ability to find solutions to the problems we face in our everyday life. Thinking in all its forms and reflecting on different behaviors helps students get to know themselves as well as others. Hence students gradually realize that their desire for independence and freedom has to be reconciled with responsibility and consideration toward others. Given the characteristics of adolescents, i.e. their egocentrism, the task is not easy, and it may take many years of patience and caring before most adolescents come to understand that their freedom ends where that of others begins.

E for empathy: Empathy is a vital quality in all relationships and especially in those that constitute the helping and caring professions. Empathetic teachers try to put themselves in their students' shoes. This attitude enables them to be flexible and adapt their expectations to their students' needs. Teachers who are empathetic present a positive role model for the development of empathy in their students. Moreover, the encouragement of empathy in students is associated with a cooperative spirit. It has a positive impact on the sense of trusting, belonging, connecting, caring, and feeling worthy in students. Such feelings are the opposite of those found in classrooms that are dominated by social isolation, bullying, teasing, ignoring, aggression and disrespect among students.

A for activity: The roots of the role of activity in learning and development are established in Piaget's (1969) theory of intellectual development. When teachers want to ensure that their students benefit and learn, they try to involve them in the learning process. They invite their students to actively participate in class and provide them with their vital support to learn. Research has repeatedly shown how students' active involvement helps their motivation in the classroom. Even physical activity should be included, whenever possible in academic classrooms, especially with adolescents, since, as reported by Sprenger (2005), movement strengthens brain structures such as memory abilities. Moreover, although adolescents are not generally thrilled about attending classes, they may like and even thrive in a classroom environment where they 
are allowed and encouraged to become actively involved in their learning through communicative activities, debates, oral presentations, and so forth.

C for choice: Most teachers find that providing their students with choice enhances their motivation to learn and participate in the classroom. A common complaint among students is that they have no choice but to do as they are asked both at school and at home. This usually has a negative impact on getting them to become responsible. When teachers allow their students to make choices within realistic parameters students seem to be more enthusiastic and involved in their learning. Their initiative to speak up, to ask questions, and to develop ideas seems to increase as well. This is not surprising since everyone has the need to find personal meaning in what they do. Finally, it is not surprising that being allowed some choice leads to feelings of being important and worthy while it enhances learning since choice is associated with crucial concepts such as autonomy, motivation and initiative.

$\mathbf{H}$ for harmony: Harmony in the classroom refers to a safe, stimulating, and positive climate where students feel emotionally secure, respected by their teacher as well as their peers and encouraged to communicate and exchange opinions, ideas, and concerns. In harmonious classrooms students find many opportunities to interact, communicate, and cooperate with others. As they progress intellectually they are helped to develop trust and learn to attain higher levels of social and emotional maturity. In classrooms where this does not happen, they may be bound to remain dependent and at risk of developing various forms of problem behaviors.

Think about it: We were all once adolescents. Do you remember how specific situations felt? For example, how did you feel when faced with rejection or the betrayal of a friend or when dealing with conflicts with authority? What were your dreams?

Although a gap exists between adolescents and adults, adolescents are, in fact, in real need of significant and durable relationships with adults that they can trust and use as role models to guide them in their struggle to develop their identity. Do you feel comfortable being a role model?

Tape record one of your lessons. A few days later listen to the way you interact with your adolescent students. Can you find examples of T.E.A.C.H.

\section{GLOSSARY}

- identity versus diffusion: Originally called "role confusion" by Erikson, this refers to the crises in which adolescents try to reconcile their need to define who they are among many different and confusing roles or identities

- egocentric: a tendency in adolescents to focus on themselves and believe that their thoughts and feelings are unique and more significant than those of others

- magical thinking: thinking that springs from feelings and imagination rather than from logic and rationality 
- trust: an essential quality needed for the establishment of a positive and significant relationship between two individuals

- rapport: a communication process that is marked by harmony and affinity

- compromise: one of Piaget's logical principles that refers to the ability to reconcile two different aspects or extremes

- give and take: two opposite actions that, when coordinated, lead to mutual understanding and harmony.

\section{References}

1. Dolto, F. La cause des adolescents, Paris: Pocket. 1997

2. Douvan, E. \& Adelson, J. The Adolescent experience, New York: Wiley, 1966

3. Elkind, D. The Child and Society, N.Y: Oxford University Press. 1979

4. Erikson, E.H. Identity: Youth and crises, New York: Norton. 1968

5. Erikson, E.H. Childhood and Society, N.Y: Norton. 1963

6. Hoffman, M.L. Development of moral thought, feeling and behavior. American Psychologist, 1979, p. 34

7. Jacobs, J.E., Lanza, S., Osgood, D. W, Eccles, J.S., \& Wigfield, A. Changes in children's self-competence and values: Gender and domain differences across grades one through twelve. Child Development, 509-527, 2002

8. Mussen, P., Eisenberg-Berg, N. Roots of Caring, Sharing and Helping. San Francisco: W.H.Freeman. 1977

9. Piaget, J. The Psychology of Intelligence. Totowa, N.J. Littlefield, Adams,1966

10. Piaget, J. and Inhelder, B. The Psychology of the Child. New York: Basic Books, 1969.

11. Rhodes, J.E. \& Roffman, J.G. Nonparental adults as asset builders in the lives of youth. In Sprenger, M. (2005) Inside Ami's Brain. Educational Leadership. Vol. 62 , no. 7 , pp. 28-32 